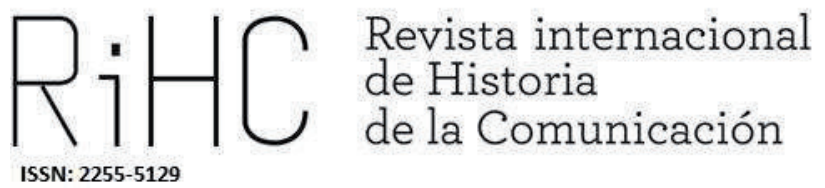

\title{
LA EVOLUCIÓN DE LA PRODUCCIÓN ORIGINAL DE LA PLATAFORMA DE PAGO HBO
}

The evolution of the original production of the paly television network $\mathrm{HBO}$

DOI: http://dx.doi.org/10.12795/RiCH.2021.i17.12

Recibido: $30-9-2021$

Aceptado: 7-12-2021

Álvaro Linares-Barrones

Universidad Pablo de Olavide (Centro Universitario San Isidoro), España

alinaresbarrones@centrosanisidoro.es

ORCID (D) 0000-0002-9801-7104 
Resumen: Cuando el servicio de televisión de pago HBO debutó en el otoño de 1972, jamás se llegó a imaginar que su futuro iba a ser tan prometedor. En estas cuatro décadas, la cadena se ha convertido en una institución con un servicio que ha cambiado la televisión, ha reconfigurado la industria del cine y ha avivado el entretenimiento en el hogar. Gracias a la evolución de su producción original, $H B O$ ha creado un contenido audiovisual que subvierte la narrativa televisiva y los géneros más tradicionales dando paso a una nueva forma de contar historias donde la identificación con el espectador es mayor.

Palabras clave: Televisión por cable, producción televisiva, programa de televisión, entretenimiento, contenido de programa.

\begin{abstract}
When the pay television network HBO started in the autumn of 1972, it was never believed that its future would be so promising. In these four decades, the cable television has become an institution with a service that has changed television, has reconfigure film industry and has simulated home entertainment. Thanks to the evolution of its original production, $H B O$ has made audiovisual content that changes television narrative and traditional genres. This fact has cleared the way to a new method of telling stories where the identification with audience is greater.
\end{abstract}

Keywords: Cable television, television production, television programmes, entertainment, programme content.

\title{
Introducción y metodología
}

Desde finales de los años 80, la televisión de Estados Unidos se ha transformado, pasando de una estricta rigidez de un oligopolio de tres cadenas (CBS, NBC y $A B C$ ) a una auténtica fragmentación del mercado con multitud de canales disponibles. En este escenario, el canal de televisión de pago HBO (Home Box Office) se ha convertido en un ejemplo de televisión de calidad a través de las normas que el propio medio le imponía, como, por ejemplo, las restricciones de adquisión de los productos audiovisuales o las estrictas regulaciones de emisión por cable. Con la sede en Nueva York, desde su aparición en 1972, ha sumado suscriptores cada década enfrentándose a numerosos retos y realizando estrategias comerciales en busca de contenidos exclusivos y originales.

Por otro lado, a finales del siglo XX se produjo una nueva etapa en la ficción televisiva estadounidense que se ha ido imponiendo en el resto del mundo: la tercera edad dorada de la televisión. A través de nuevos contenidos donde la calidad es el principal sustento, las cadenas de suscripción y/o pago, como es el caso de HBO, comienzan a ofrecer nuevas historias más heterogéneas con personajes que van más allá de los estereotipos habituales. Este nuevo modelo, es representado en la producción original creada por HBO, la cual ha ido evolucionando a medida que ha ido creciendo la plataforma de pago. 
HBO es una cadena pionera tanto en la implantación y desarrollo de la televisión de pago, como en la creación de contenido audiovisual. Su importancia radica en el momento que se convierte en parte esencial de los hogares de todos los ciudadanos idenpendientemente del lugar en el que se encuentren. Este estudio sobre la evolución histórica de la plataforma HBO, haciendo mayor énfasis en su producción original, se debe a su relevancia como un claro referente del proceso de transformación de la estructura del medio audiovisual. Es decir, forma parte de aquellos agentes que rompen progresivamente con las ventanas de explotación tradicionales, y que se instauran en todo el mundo con nuevos modelos de negocio, cambiando completamente las reglas del escenario televisivo.

En cuanto a la metodología empleada, se ha utilizado un método de investigación cualitativo, en concreto una revisión bibliográfica. Orientándose y condensándose la información, el estudio se presenta como un análisis descriptivo que facilita una mirada reflexiva de lo que ha supuesto la plataforma de streaming HBO para la industria audiovisual. El propósito es elaborar una información completa y exhaustiva del contenido propio de la plataforma de pago, utilizando diversas referencias bibliográficas caracterizadas en la materia y seleccionando aquellas fuentes que han investigado el fenómeno de HBO desde diversos campos, como su producción (DeFino, 2014), su historia (Mesce, 2015), sus formatos o géneros (Cascajosa, 2006) o su modelo de negocio (Celeya, 2014). Gracias a esta revisión histórica de la cadena HBO, se construye un texto descriptivo como base teórica para investigaciones futuras relacionadas con la evolución de las plataformas de streaming que imperan hoy en día como principales medios de consumo audiovisual.

\section{El modelo de negocio}

En las dos últimas décadas, se han creado nuevos hábitos de consumo audiovisual cambiando por completo las ventanas de explotación tradicionales dando lugar a diferentes modelos de negocio. El consumo del audiovisual en la red ha derivado en una democratización total del entretenimiento (Monzoncillo y Sendrá, 2010) que a su vez ha generado un nuevo usuario digital basado en el aquí y ahora. Se trata de un espectador, que, de forma individual y personal, participa, produce y se descarga sus propios contenidos en la red dando paso a nuevos formatos de visualización (Del Pino y Aguado, 2012). Son compañías que ofrecen un catálogo audiovisual muy extenso tanto de series como de películas que pueden ser consumidas en streamimg, y que están provocando 
que los llamados servicios Over The Top ${ }^{1}$ (OTT) se conviertan en un elemento estratégico para las empresas de entretenimiento y para las cadenas de televisión tanto en abierto como por cable (Huerta y Domínguez y Sanz, 2011). Este modelo de negocio cada día se expande más y se denomina long tail, que consiste en "vender menos de más, sobre una amplia gama de productos especializados" (Osterwalder y Pigneur, 2011: 67). Se trata de un modelo que continuamente debe renegociar los derechos de exhibición de los contenidos audiovisuales por Internet en función de las directrices de las distribuidoras en cada país (Ojer y Canapé, 2012).

La nueva distribución online de contenidos genera un panorama para la comunicación digital donde se van consolidando "modelos de negocio compatibles con la cultura de consumo del nuevo espectador-internauta" (Izquierdo, 2012: 385). Las plataformas online de productos audiovisuales, como HBO, provocan que el mercado de la distribución de contenido se dibuje en torno a tres modelos de negocio que a su vez suponen tres fuentes de financiación (Izquierdo, 2012 y 2015):

- Micropago: pago por unidad consumido en directo y en el momento que es solicitado, o por medio de venta electrónica, en el que el público adquiere el contenido en propiedad.

- Cuota mensual: visionado ilimitado del contenido por una cantidad económica concreta estipulada previamente por la compañía.

- Modelo dependiente de publicidad: acceso gratuito sustentado por anunciantes, un atractivo para el espectador que se asemeja a lo que se puede encontrar en el escenario offline.

En este nuevo sector de distribución online de contenidos, HBO destaca por operar con la cuota mensual o vídeo por suscripción. Se trata de un modelo de negocio en el que se ofrece un contenido atractivo al mismo tiempo que supone una fórmula de monetización muy útil para el usuario, con ofertas de contenido televisivo a través del reproductor online en streaming, sobre todo programas de producción propia (Guerrero Pérez, 2011). Además, este modelo de negocio utilizado por muchos ámbitos de la cultura (música, editoriales, videojuegos) ofrece la posibilidad de trabajar con una base de clientes fija en un tiempo concreto $y$, por tanto, ser una fuente de ingresos segura cada mes (Celaya, 2014). Por ello, HBO distribuye en línea los contenidos audiovisuales que la audiencia activa puede consumir masivamente, $y$ al instante, por medio del pago por suscripción que le ofrece el acceso al catálogo completo. Este tipo de plataforma ayuda a monitorizar los gustos del consumidor por medio de los filtros y a la creación por parte de la cadena de una producción propia adaptada a las necesidades de sus suscriptores (Bonaut Iriarte, 2016). Hay que señalar que HBO ofrece un consumo de contenidos en modo offline, es decir, que todas sus series o capítulos no están 
disponibles de manera inmediata por el riesgo a perder clientes, como sí es el caso de su principal competidora Netflix.

\section{El secreto de la narración}

Con una importante línea argumental, HBO siempre ha hecho sus propias historias en base a lo que sus suscriptores han demandado. En el documento HBO A Brand Story ${ }^{2}$, que referencia el autor Santaella (2016) en su publicación, se indica que la marca a la hora de realizar producciones propias debe "empujar los bordes de la creatividad y la innovación hacia nuevos estándares de excelencia, llevando a las alturas la experiencia del espectador" (p. 50). Además, se añade que la voz de HBO es:

- Auténtica: nunca elige estilo por encima de la sustancia.

- Retadora de pensamientos: reta las creencias aceptadas.

- Arrojada: constantemente explora nuevos territorios.

- Valiente: siempre está dispuesta a tomar riesgos.

- Ingeniosa: fusiona humor y encuentra la verdadera naturaleza de la situación.

- Apasionada: rechaza el compromiso que estanca.

HBO siempre ha sido una cadena que ha trabajado de forma significativa sus relatos, contando historias por medio de un storytelling que deja ver "la pasión de HBO por la creatividad independiente, la calidad artística de la escritura y la excelencia de la producción" (Santaella, 2016: 50). Sus historias desafían al público intelectualmente y reconstruyen los géneros más tradicionales. Por ello, la narrativa desarrollada por HBO se refleja en la producción de series de calidad con la atención puesta en la relación con el espectador, parte fundamental de la misión y de la visión de la marca.

A través de este storytelling, HBO desarrolla una especie de entropía narrativa. Es decir, al igual que en la mayoría de las historias, los personajes dentro de la narración intentan crecer y cambiar, mientras que el mundo y sus obligaciones lo hacen también, y en el transcurso de esto, aprenden cosas importantes sobre ellos mismos. En relación con esta entropía narrativa, al igual que muchos relatos, la mayoría de las historias que desarrolla HBO utilizan la muerte como fuente de significado. La muerte como una entrada a la vida eterna, como un sacrificio al bien mayor o como una liberación de bienvenida. El drama de Six Feet Under (2001-2005) es un ejemplo que lo refleja a la perfección (Akass y McCabe, 2005).

2 Manual de branding de la compañía en el que viene desarrollado la filosofía de la empresa. 
Por otro lado, un aspecto destacado dentro de la narrativa desarrollada en la producción original de $\mathrm{HBO}$ son sus finales controvertidos que frustran las expectativas de un cierre satisfactorio para todos. Los espectadores de Sex and the City (1998-2004) fueron divididos en partes iguales, como en el caso de The Sopranos (1999-2007). Otras ficciones con menor alcance mediático, como el drama Big Love (2006-2011) y las sesiones de psicoterapia que dieron vida a la serie In Treatment (2008-2010, y en proyecto de volver en 2021), terminaron también de una manera muy reflexiva (García Martínez, 2010). Mientras tanto, series como Carnivàle (2003-2005) y el drama histórico Rome (2005-2007) co-producido con la BCC y la Rai (Radiotelevisión Italiana) como estrategia de internacionalización, se vieron obligadas a comprimir tramas y abandonar aquellas historias de menor importancia después de sus cancelaciones prematuras (Mesce, 2015). Pero uno de los finales más recientes y controvertidos ha sido el de la exitosa serie Game of Thrones, el cual dejó al público dividido, habiendo un mayor porcentaje hacia el descontento (El País, 2019). En general, se trata de miles de historias que han acabado con finales significativos donde el acumulado de la historia solo puede realizarse plenamente en su resolución. En su producción original HBO quiere mostrar que los personajes se enfrentan frecuentemente a la verdad subyacente como ocurre en la realidad. Es decir, en la vida real como en la narración, las historias están llenas de incertidumbre con relaciones que van y vienen, y propósitos que finalmente se desvanecen con mucha más frecuencia que se consiguen.

\section{La producción original}

Teniendo una presencia en más de 150 países en todo el mundo, HBO tiene una programación compuesta por estrenos de películas, ya exhibidas en las salas de cine, así como series y películas de producción propia, contenidos deportivos, en concreto peleas de boxeo, conciertos y documentales, y posee otros canales como HBO2, HBO Plus o HBO Family. La red siempre ha sido considerada como la principal proveedora de películas sin censura ${ }^{3}$ (temas tabúes como el sexo, la violencia o asuntos

\footnotetext{
3 Las películas estadounidenses estaban regidas por el código Hays, creado por la asociación de productores cinematográficos de Estados Unidos (MPAA), que determinaba lo que se podía ver o no en pantalla desde 1934 hasta 1967. Se trataba de un código muy estricto y menos naturalista donde el sexo, los crímenes, el alcohol, la religión e incluso el vestuario, eran temas duramente regulados. Luego, se instauró la clasificación por edades de la MPAA (vigente actualmente) en la cual los contenidos anteriores son abiertamente expuestos para los adultos con calificación para los menores (Durán Manso, 2016). En cuanto a la censura en la televisión por cable, está regulada sobre todo por la Ley General de Telecomunicaciones de 1982, y el Consejo Nacional de Televisión de 1989. En el caso de HBO, al no ser un servicio público, tiene mayor libertad en la exhibición de sus contenidos, pero debe cumplir, como impone el Consejo, con unas normas generales en base a la violencia excesiva, la truculencia, la pornografía y la participación de niños o adolescentes (Barrios Socías, 1998).
} 
controvertidos), programación deportiva y comedias especiales en vivo. A través de su producción original, en concreto sus célebres series, ha reflejado y ha influido en la cultura gracias a las nociones convencionales de realidad y política.

\subsection{Inicios de la producción original: el deporte}

Cuando se habla de la producción original de $\mathrm{HBO}$, se relaciona estrechamente con los guiones de dramas y comedias. Pero el grueso de la programación producida por la cadena en sus comienzos estaba en el área del deporte. De la mano de Michael Fuchs, abogado de formación, HBO comenzó a trabajar en la producción propia. Se pretendía conseguir que la empresa pasara de una simple plataforma de exhibición de películas a una organización polifacética de mayor alcance. De 1973 a 1975, el servicio ofreció un promedio de más de 200 eventos deportivos al año. Debido a las regulaciones antisiphoning ${ }^{4}$ de la agencia estatal de Estados Unidos, la FCC (Comisión Federal de Comunicaciones), se hizo casi imposible adquirir importantes licencias de franquicia. Por tanto, las ofertas del servicio eran diversas e iban desde ocasionales partidos de baloncesto hasta el hockey y la natación (Mesce, 2015). Series dedicadas al deporte que han estado presentes en toda la historia de HBO: HBO World Championship Boxing (1973-2018), Wimbledon Tennis (1975-1999), Inside the NFL (1977-2008); y decenas de partidos de la NBA, NHL y los juegos de los New York Yankees.

Pero incluso después de que las restricciones fueran levantadas en 1977 por decisión de la FCC tras la victoria de HBO en el pleito interpuesto por la compañía ${ }^{5}$, la empresa no contaba con los recursos suficientes para invertir en los deportes más populares de manera regular, por lo que decidió empezar a concentrar su atención en áreas deportivas que no se emitían con tanta frecuencia en televisión, como el campeonato de tenis de Wimbledon. Del mismo modo, fueron capaces de convertirse en The Home of Boxing, ya que era un deporte sin emisión en ningún canal regularmente. Poco a poco, HBO se convierte en la fuente de registro para muchos de los combates más significativos de la historia del boxeo: Holmes-Cooney en 1982, y Hagler-Hearns en 1985 (DeFino, 2014).

El evento deportivo más importante de la cadena fue uno de los campeonatos de boxeo de mayor peso y más esperados de la historia del deporte. El día 30 de septiembre de 1975, HBO se convierte en la primera cadena de televisión en transmitir su señal por satélite con la distribución del combate de boxeo Thrilla en Manila entre Muhammad Ali y Joe Frazier (Edgerton y Jones, 2008). Este combate, ofrecido en directo, se convirtió

4 Reglas de emisión que protegían a las cadenas de televisión públicas (DeFino, 2014: 46).

5 En 1977, HBO ganó su pleito anti-siphoning en contra de la FCC, que dio a la industria del cable de pago el acceso abierto a las películas de Hollywood, series originales y programación deportiva nacional, lo que provocó un aumento significativo del valor de los sistemas de cable (De Fino, 2014: 59). 
en una de las fortunas de HBO de toda su historia, estableciendo al mismo tiempo su reputación como una fuente de élite en relación al boxeo, y demostrando perfectamente que el satélite brindaba una tecnología con una imagen impecable en vivo (Parsons, 2003). Tras el éxito de la cadena en materia de deportes, se creó el canal HBO Sports, una de las claves de la programación de la compañía.

\subsection{Más allá de los eventos deportivos: las primeras producciones originales}

Dejando a un lado la categoría de deportes, la primera oferta original de HBO, que se estrena solo cuatro meses después de la emisión inaugural del canal, fue un concierto de música protagonizado por las cabezas de cartel Tina Turner y Barbra Streisand (Mesce, 2015). En aquellos primeros años, la empresa tenía un problema de programación táctica: solo estaban disponibles un número reducido de películas. Como se ha comentado antes, la FCC imponía unas restricciones sobre qué películas podían ser transmitidas en la televisión de pago (no se encontraban títulos de menos de dos años o más de diez). Además, los distribuidores de películas todavía veían con recelo el servicio naciente, siendo reacios a conceder licencias de títulos importantes a la televisión de pago.

Por necesidad, HBO debía encontrar algún producto para complementar su programación de cine por lo que comienza a jugar con los diferentes formatos disponibles. La cadena de pago realizó sus propias producciones para el cine con costes más económicos y fuera de la cultura propia de los estudios de Hollywood. Esto recibía el nombre de HBO Films y su producción inaugural fue The Terry Fox Story (1983), considerada la primera película producida por una televisión de pago. Se trataba de un film biográfico sobre un corredor canadiense que realiza una carrera por todo el país de Canadá para recaudar fondos y concienciar a la sociedad sobre la investigación del cáncer (Leverrete, Ott y Buckey, 2008).

Por otro lado, HBO empieza a apostar por una nueva forma de documental televisivo basada en los elementos narrativos del cine, la literatura y el teatro, contando de cerca las historias personales de gente común en circunstancias extraordinarias. Evitando la objetividad a la hora de empatizar o identificarse con el drama convencional, fue un género que le dio credibilidad en sus principios como productor. En 1979, el servicio estrenó una serie documental de seis partes llamada Times Was que resultó ser una mezcla de entretenimiento y educación que dio lugar a dos series similares, Yesteryear (1982) y She's Nobody's Baby (1981) (Mesce, 2015). 


\subsection{La comedia como principal género en la producción original}

Uno de los géneros más explotados por HBO al comienzo de la elaboración de su propia producción fue la comedia. Aunque se tiende a asociar HBO con dramas, la red ha producido una programación cómica durante un período de tiempo más largo que el de cualquier otro género. De esta manera HBO, utilizando una de las que sería su seña de identidad, mostraba lo que la televisión pública y más tradicional no podía: contenido con lenguaje obsceno, desnudos y temas controvertidos. Además, desarrolló nuevas formas de comedia como el sketch (con el surrealista Mr. Show with Bob and Dave, 1995-1998), los talk show (con temas orientados a la política de Dennis Miller Live, 19942002; y Real Time with Bill Maher, 2003-presente) y la comedia de situación (con sátiras posmodernas como The Larry Sanders Show, 1992-1998; y Curb Your Enthusiasm, 2000presente). La comedia fue la oruga de HBO que más tarde se convertirá en mariposa, ayudando a la red a transformarse de un servicio de cine y deportes a un estándar de oro de la producción original (DeFino, 2014).

Hasta principios de 1980, la red no se introduce en el negocio de este género, que coincidirá con el lanzamiento del satélite. En víspera del Año Nuevo de 1975, se emitió la primera entrega de una serie mensual de stand-up Ilamada On Location ofreciendo a HBO una oportunidad para demostrar el tipo de producción provocativa y sin censura que hacían. En poco tiempo HBO se convierte en un nuevo hogar para la comedia standup porque, al igual que el boxeo, estaba disponible en la plataforma a muy bajo coste y tenían lo que la compañía llamaba "hard-ticket value" (Sullivan, 2010: 87).

Las comedias stand-up tomaron mayor fuerza con la presencia de numerosos cómicos conocidos como Phyllis Diller o Eddie Murphy. Después de varias producciones cómicas como Not Necessarily the News (1983-1990), The Kids in the Hall (1988-1995) o Comic Relief (1986), HBO comenzó a buscar proyectos que identificaran aún más a la cadena con la comedia de calidad. Un reflejo de ello fue la miniserie Tanner '88 (1988), una aguda crítica a los políticos creados a través de los medios de comunicación. Esta producción demostraba el compromiso de HBO en hacer una televisión que difuminara la línea entre realidad y ficción. También, dejaba ver varias características ligadas hacia la forma documental, como la técnica de producción fly-on-the-wall, filmando entrevistas por medio de una narración estentórea que dejara ver una observación objetiva de los acontecimientos (Edgerton y Jones, 2008).

Mientras Tanner '88 representaba significativamente una evolución en la producción original de la cadena, muchos críticos y suscriptores no comprendían el efecto que generaba la creencia sobre lo real/falso en estas narraciones. Por ello, y en busca de un mayor éxito, HBO evoluciona más allá de la cuarta pared y se integra perfectamente en el modo de contar historias. La primera serie encargada de conseguir este efecto fue The Larry Sanders Show (1992-1998), un producto que entrelaza dos narrativas: la 
"pública", transmitida a través del programa de entrevistas nocturnas de ficción llamado The Larry Sanders Show, y la "privada", un pequeño drama detrás del escenario que mostraba la lucha del protagonista con los problemas diarios denominado The Larry Sanders Meltdown (DeFino, 2014). El estilo de The Larry Sanders Show iba más allá de las restricciones retóricas del falso documental, al mismo tiempo que disipaba la línea entre la realidad y la ficción. La serie dibujaba una estética específica para cada escenario revelando diferentes niveles de la realidad. El propio programa de entrevistas nocturnas, The Larry Sanders Show, se grababa en colores vivos, live on tape, utilizando tres cámaras fijas, aplausos e iluminación y sonido de estudio. Sin embargo, las escenas de The Larry Sanders Meltdown se grababan utilizando una sola cámara itinerante y una película granulada que producía colores apagados a partir de la luz ambiental (Leverrete, Ott y Buckey, 2008). En general, The Larry Sanders Show servía como prueba de que la plataforma HBO podía hacer espectáculos únicos y excepcionales. Aunque no fue un éxito, la serie consiguió una buena crítica llegando a recibir un total de 56 nominaciones Emmy, incluida la de mejor serie de comedia en sus seis años de vida, y premios importantes de la televisión entre los que se encuentran los Emmy Awards o los Golden Globe Awards (IMDb, s.f.). Además, la producción se posicionó en el puesto 38 de la TV Guide's 50 Greastest TV Shows of All Time, convirtiéndose en la única serie de comedia de HBO en estar presente en esta lista (CBS News, 2002).

Más tarde, la cadena de pago continuó haciendo comedias basadas en los temas centrales de The Larry Sanders Show: la cultura de la celebridad y la estrecha línea entre la realidad y la ficción. Programas como Arlis\$ (1996-2002), Tenacious D (1997-2000) y Entourage (2004-2011) se mofaban del concepto de popularidad rompiendo el mundo real de los deportes, la música, el cine y los negocios. Este legado de The Larry Sanders Show, donde mejor se visualizó, fue en la serie de comedia con mayor duración de la historia de HBO: Larry David's Curb Your Enthusiasm, estrenada en 2000 y vigente en la actualidad. Curb es un excelente ejemplo de la clase de comedia americana que se dio en el 2000 de la mano de la plataforma, frecuentemente temporal y potencialmente ofensiva para casi cualquier grupo minoritario que se nombre en el programa (Fernández y Aguado, 2013).

Por otro lado. se desarrollaron otras series de comedia que no se inspiraron en The Larry Sanders Show como Sex and the City, The Comeback (2005-2014), Veep (2012-2019), Divorce (2016-2019) o Girls (2012-2017), ligadas más hacia una línea dramática (comedias dramáticas) y favoreciendo a las tramas y a los arcos de personajes. En el caso de la serie Sex and the City, fue considerada una de las principales obras originales de HBO posicionando a la cadena en un lugar destacado en la mente del consumidor (Rubio Hernández, 2014). 


\subsection{Las ficciones dramáticas, las miniseries y los documentales}

El storytelling dentro de la gran producción original de la cadena empieza a visualizarse en especial en el género dramático. En general, en esta categoría la plataforma desarrolló un importante número de programas de calidad "cada vez más arriesgados y satisfactorios a nivel narrativo, estilístico y temático" (Cascajosa, 2006: 25). La cadena de pago va implantando a través de estas ficciones unos patrones formales que cumple en la mayoría de sus producciones originales otorgándole un sello propio. Todos los dramas de HBO tienen en común ciertos elementos que están fuera del propio relato. Uno de los más utilizados es "la radical modificación de la estructura inicial del capítulo" (Cascajosa, 2006: 32). También, destaca la inexistencia de un previo resumen de los capítulos anteriores y el comienzo directo con el openning o con la parte inicial del nuevo capítulo (mayor esfuerzo de recuerdo por parte del espectador). Otro elemento son los créditos finales o de apertura de los capítulos, en concreto se destacan el de la serie The Sopranos donde se utilizaba una música distinta en cada crédito final o The Wire (20022008) donde se utilizaba la canción Way Down in the Hole del autor Tom Waits como inicio, pero interpretada por un artista distinto en cada temporada de la ficción ${ }^{6}$.

En los comienzos de la producción de obras de corte dramático, HBO triunfó con numerosas producciones como Philip Marlowe (1983-1986), Private Eye (1983-1986) o la serie de antología Tales from the Crypt (1989-1996), pero sin llegar a posicionar a la cadena en un lugar preferente dentro de la industria televisiva. A principios de 1990, se llevaron a cabo producciones de documentales sobre la conciencia social, miniseries y películas sobre temas como el SIDA (Common Threads: Stories from the Quilt, 1989), malversación corporativa (Dead Ahead: The Exxon Valdez Disaster, 1992; Barbarians at the Gate, 1993), y los problemas de las clases bajas (I Am a Promise: The Children of Stanton Elementary School, 1992; Gang Wars: Bangin in Little Rock, 1994). Se trataba de producciones que suponían una oportunidad para desarrollar los puntos de la trama y los personajes. Una manera de enlazar la vida cotidiana con los motivos y temas más importantes de las historias, y vincularlos de nuevo a la narración principal (Carter, 1992). Pero el punto de inflexión vino de la mano del desarrollo de series con personajes más continuos, y la primera serie de producción propia con dicho elemento fue $\mathrm{Oz}$ (1997-2003), creada por Tom Fontana. El lanzamiento de esta serie dramática de una hora de duración fue la producción a partir de la cual se comienzan a crear contenidos de mayor éxito dentro de la cadena de pago, desarrollando una producción original que comienza allanar el camino hacia la edad de oro de la plataforma en los años 2000 (Edgerton y Jones, 2008).

6 Por ejemplo, la canción fue interpretada por autores como el grupo de gospel The blind boys of Alabama (temporada 1), el grupo The Neville Brothers (temporada 3) o Stive Earle (temporada 5) que también actuaba en la serie (Calvo, 2021). 
El ejemplo más relevante fue la serie The Sopranos, estrenada en 1999, que llegó a conseguir una destacada crítica y logró numerosos premios Emmy: 21 estatuillas, dos de ellas en la categoría de Mejor Serie de Drama (IMDb, s.f.). El creador de la serie, David Chase, llegó a HBO con la presentación de la ficción después de haberlo intentado en otras cadenas. Este hecho le permitió más tiempo y libertad a la hora de desarrollar los detalles visuales y narrativos de la serie gracias a la ausencia de restricciones de contenido dentro de la cadena de pago. The Sopranos fue una serie oscura y desordenada, llena de frustraciones y expectativas insatisfechas, en la que nada se puede reducir a un solo sentido y nadie es redimido (Lavery, Howard y Levinson, 2011). Además, en ella se inició la figura del antihéroe de la mano del protagonista Tony Soprano, un personaje que desempeña algunas características propias del héroe tradicional, pero que carece de otras por tener las virtudes y defectos de una persona mortal (Onandia, 2013). La serie tuvo éxito más allá de la televisión por cable y fue capaz de atraer críticas muy similares a las de otras producciones de prestigio de grandes productoras, a pesar de estar solo disponible para los suscriptores de pago (Polan, 2009).

Otros ejemplos de series dramáticas que destacaron, fueron las producciones de Six Feet Under y de The Wire en 2002. Esta última no fue capaz de superar los datos de audiencia que consiguió la serie The Sopranos, pero en el campo de la crítica consolidó la reputación de HBO como una red de producción de calidad (Lasierra, 2012). En el caso de Six Feet Under, la construcción de los personajes era muy compleja ya que el entorno de la serie exigía un elenco que contemplara profundas cuestiones metafísicas y ontológicas, particularmente en relación al tema de la muerte: qué temen o cómo se transforman (Akass y McCabe, 2005). A partir de 2010, se realizaron producciones propias de gran éxito como el drama de misterio The Leftlovers (2014-2017), o la serie de antología True Detective (2014-2019) la cual contiene elencos y escenarios distintos en cada temporada, destacando en la primera la presencia de actores cinematográficos de renombre como Matthew McConaughey y Woody Harrelson (Mesce, 2015).

En general, los dramas de $\mathrm{HBO}$ siempre se han caracterizado por contener una narrativa innovadora que ha conseguido diferenciar sus series de las de la competencia buscando la hibridación de temáticas desde las historias reales de los personajes: mezcolanza de tramas familiares (el crimen, la violencia, la pobreza) y genéricas (la reforma de la prisión o la huida en Oz; la destrucción del gángster en The Sopranos; o la corrección de los errores de la sociedad en The Wire).

\subsection{La producción infantil}

Otro campo donde la cadena de pago ha desarrollado su programación original son las producciones infantiles. En 1978, como regalo de Navidad a sus suscriptores, HBO ofreció Emmet Otter's Jug-Band Christmas, una creación de títeres del maestro Jim 
Henson ${ }^{7}$. Se trataba de un cuento que narraba las vacaciones de un grupo de criaturas musicales que entretenía tanto a los niños como a sus padres. Un éxito que condujo a Jim Henson a trabajar con HBO unos años más tarde. En 1983 creó uno de los verdaderos clásicos de la producción infantil, a la altura de Sesame Street Muppets, el largo espectáculo The Muppet Show: Fraggle Rock (1983-1987). Se emitió durante cinco años en HBO para aparecer más tarde en los televisores de todo el mundo, asentando las bases de la producción original dentro de la cadena a mediados de los 80 (Pepper, 2019).

A partir de este momento, HBO creó un trabajo dedicado no solo a los niños (como Braingames, 1983-1985; y Happily Ever After: Fairy Tales for Every Child, 1995-2000) sino también a los adolescentes. En este último caso, eran espectáculos que iban desde documentales hasta docudramas inspirados en historias reales de la vida diaria, como el SIDA o las enfermedades de transmisión sexual (And the Band Played On, 1993) hasta la homosexualidad (When I knew, 2008) y el suicidio adolescente (Boy Interrupted, 2009). Por otro lado, también había programas de dibujos animados como Mr Show with Bob and Dave (1995-1998) y Tracey Takes On... (1996), presentando a personajes neuróticos y egocéntricos. Por ello, desde las series de animación para los más pequeños hasta los dramas para los adolescentes, HBO se convirtió en una cadena con una de las programaciones infantiles más respetadas en el mundo televisivo (DeFino, 2014).

A principios del año 2000, la empresa comienza a reducir sus contenidos de producción original familiar hasta el año 2015 cuando decide reiniciarse en este género a través de su contrato de cinco años con Sesame Workshop. En dicho acuerdo se creó la serie de televisión educativa para niños Sesame Street (2016) -correspondiente a su 46a temporada- que combina acción en vivo, comedia de bocetos, animación y títeres. Se caracteriza por contener imágenes con títeres de estilo burlesco y metareferencial, y por incluir cortometrajes con humor y referencias culturales (Pallotta, 2019).

\subsection{La proliferación del género fantástico}

Cerrando la producción original de HBO, la cadena de pago ha estado desarrollando series que conectan con la identificación de los espectadores más jóvenes a través de su programación. Se trata de la proliferación de series de terror y fantasía a partir del estreno de True Blood (2008-2014), un drama de vampiros basado en las novelas góticas de Charlaine Harris ${ }^{8}$ que cautivó al público (Mellins, 2012). En la mayoría de los relatos

7 Titiritero y productor televisivo estadounidense conocido por ser el creador de The Muppets. Estuvo nominado a un premio Óscar y ganó los premios BAFTA y Emmy por su trabajo como director de programas musicales o de variedades.

8 Escritora estadounidense de bestsellers dedicada al género de misterios durante más de veinte años. 
fantásticos producidos por la plataforma de pago, temas como la belleza, el horror, el amor y el dolor se llevan hasta el extremo.

La serie más exitosa hasta la fecha de la cadena en este género ha sido Game of Thrones, que expresa significativamente la esencia de HBO. La violencia y la misoginia de su universo pueden resultar muy familiar para los fans de Deadwood (2004-2006), y la representación de las jerarquías sociales a través de las historias interconectadas del amplio elenco de personajes, se asemeja a la serie The Wire. Además, Game of Thrones analiza el papel de la clase, el género, la raza y la religión por medio del objetivo principal de los protagonistas: conseguir o mantener el poder (Linares Barrones, 2021). Técnicamente la fantasía desarrollada dentro de la ficción se ocupa principalmente de la política, la estrategia militar y el drama interpersonal, y está muy relacionada con los juegos de rol (Lozano, Raya y López, 2013).

La mayoría de las producciones fantásticas de $\mathrm{HBO}$ se caracterizan por contener elementos sobrenaturales que reflejan el estado sociopolítico de la historia, tales como los vampiros de True Blood o los dragones de Game of Thrones. En True Blood, los vampiros están destinados a evocar el miedo hacia lo extraño utilizados como una metáfora en relación al racismo o la homofobia; y en Game of Thrones, los dragones representan las figuras que desafían la voluntad humana y la ambición como referencia al cambio climático y otras fuerzas de la naturaleza. No son objetos de fantasía sino alternativas de evasión a nuestro propio mundo de conflictos y problemas que requieren soluciones. Es decir, se trata de un mensaje en el que se pone en alza que los peligros no se van a destruir matando vampiros o dragones, sino que hay que aprender a adaptarse a las condiciones cambiantes del mundo que nos rodea (DeFino, 2014).

Otra de las series dentro de la producción original de la plataforma que refleja la utilización del género de fantasía es la ficción distópica Westworld (2016-presente). El relato se contextualiza en un parque de atracciones futurista donde gracias a la tecnología el lugar se convierte en una simulación del viejo oeste. En él, los invitados pagan por vivir unas aventuras de fantasía que en la vida real no sería posible. Al igual que ocurre en el resto de producciones originales de la cadena, la narrativa de cada personaje prima por encima de los aspectos fantásticos que ocurren en el relato (Huertas Pérez, 2018). Además, esta realidad paralela creada en la serie refleja dos fenómenos que componen a la actual sociedad de consumo imperante, el capitalismo global y la ubicuidad de las nuevas tecnologías (Olveira, 2020). Recapitulando, HBO va apostando por la fantasía y la espectacularización en sus producciones con historias más transgresoras que en cierta manera representan aspectos del mundo moderno a través de la designación de elementos fantásticos. 


\subsection{La producción original en la actualidad}

En la actualidad, los documentales y los eventos deportivos siguen siendo activos valiosos para HBO, y a esto se le suman las ficciones de corte dramático, las cuales siguen conformando la mayoría de su programación. En especial la clave del éxito de HBO es la programación serial que anima a los espectadores a volver semana tras semana, mes tras mes, al servicio. Por ello, con el fin de impulsar la lealtad del espectador, el contenido de HBO siempre se ha distinguido y diferenciado de la competencia, ofreciendo nuevos productos precursores en lo que a narrativa y género se refiere.

Desde 2018 HBO pasa a convertirse en propiedad de AT\&T, una compañía holding conglomerada multinacional estadounidense (el segundo proveedor de servicios de telefonía móvil y el mayor proveedor de servicios de telefonía fija en Estados Unidos). Tras la adquisición, el modelo de programación de HBO cambia hacia uno mucho más rentable y productivo con mayor contenido de producción original (similar al que ofrecen otros servicios como Netflix) para lograr un compromiso más fuerte con los suscriptores (Lee y Koblin, 2018). Por ello, HBO en 2018 decidió realizar cambios en la dirección de su programación empezando a interrumpir las transmisiones de boxeo después de 45 años tras un aumento de la competencia (Zarazua Abascal, 2018), y creó el servicio de televisión premium HBO Max donde poder visionar una variada programación adicional (Orgaz, 2019). Finalmente, en el año 2021 la plataforma HBO pasó a denominarse HBO Max, conglomerando todos sus contenidos en un solo servicio (Pérez Miranda, 2021).

Actualmente, la mayoría de la producción original de HBO está orientada a un público adulto a través de series (dramas y comedias), miniseries y largometrajes. Algunos de los títulos más novedosos e influyentes dentro de la plataforma son Veep (2012-2019), Silicon Valley (2014-2019), Insecure (2016-presente), Succesion (2018-presente), Watchmen (2019), Years and Years (2019), Big Little Lies (2017-2019), Chernobyl (2019), Euphoria (2019-presente), The Undoing (2020), Perry Mason (2020-presente) entre otros. Muchas de estas producciones han sido galardonadas en los últimos años en premios de alta categoría como los Emmy o los Globos de Oro, dejando ver la importancia y rigurosidad de la cadena de pago por suscripción como productora de entretenimiento.

\section{Conclusiones}

Después de cuatro décadas construida sobre las innovaciones disruptivas de cable y satélite en base al vídeo doméstico y el streaming, $\mathrm{HBO}$ se ha convertido en una empresa grande, exitosa y profundamente arraigada en los valores del mercado existente. Su 
producción original es un atributo principal para la cadena reformando de manera efectiva el concepto de televisión de calidad tanto en el drama como en la comedia a través del desplazamiento de las convenciones del género y de la ampliación de los límites de contenido y forma.

La plataforma de streaming $\mathrm{HBO}$ ha evolucionado muy significativamente en cuanto a su producción original diversificando hacia un tipo de servicio proveedor de productos con mayor originalidad y exclusividad, siendo una de las cadenas de pago más premiada hasta el día de hoy. Además, es uno de los agentes que ha roto de manera progresiva las ventanas de explotación tradicionales de los productos audiovisuales generando nuevos e innovadores modelos de negocio.

Su contenido a lo largo de los años ha estado marcado por uno de los agentes más característicos dentro la cadena, la representación de los temas más controvertidos en la sociedad, especializándose en temas que las propias cadenas generalistas no podían emitir por motivos de limitación de edad -en ciertas franjas horarias- y de financiación publicitaria. A través de una producción original con contenido explícito, la plataforma de pago ha influido y reflejado la cultura con una noción de realidad y política. Adem-as, muchas de las producciones de $\mathrm{HBO}$ han sido referentes por reflejar cuestiones sociales de vital importancia como la homosexualidad o la muerte (Six Feet Under), la violencia (The Sopranos), el sexo explícito (Oz), los conflictos bélicos (Generation Kill, 2008) o la drogadicción (The Corner, 2000), entre otros muchos asuntos polémicos.

La prorgamación de la cadena ha sido adaptada continuamente a los gustos, necesidades y deseos de una audiencia heterogénea a través de la creación de una producción propia, y ha estado marcada por la racionalización del contenido para así llegar a distinguirse del resto en el mercado (disponible utilizando cualquier tipo de dispositivo o plataforma multimedia). Además, la platafroma de streaming se ha reinventado en cada década creando producciones que han supuesto una evolución narrativa en cada uno de los géneros en los que ha estado presente: el deporte, la comedia, el drama o las producciones infantiles.

En este sentido también ha supuesto un agente precursor, ya que abandonó la clásica comedia de situación apostando por la metaficción, la autorreferencialidad y las formas del cinema-verité. También, ha conseguido asentar la necesidad de proyectar el suspense dramático (Big Little Lies) así como explotar géneros totalmente desiertos por parte de la televisión, como el caso del género fantástico (True Blood). Ha reformado otros géneros como el thriller (True Detective), y ha incorporado en sus producciones el desarrollo de una puesta en escena y estética más cinematográfica (Game of Thrones). Gracias a su narrativa, HBO va apostando por la creación de producciones con relatos más transgresores que representan las situaciones y los problemas del mundo actual. 


\section{Referencias bibliográficas}

AKASS, K. y MCCABE, J. (2005): Reading "Six Feet Under": tv to die for, Londres, Reading Contemporary Television.

BARRIOS SOCÍAS, M. (1998): "La censura en la televisión por cable". Revista Chilena de Derecho, n4, octubre-diciembre de 1998, pp. 793-825. Recuperado de https://bit.ly/3dKIIJY

BONAUT IRIARTE, J. (2016): “La muerte de la tercera edad dorada de la ficción televisiva: ¿y ahora qué?” en Nueva Revista, no158, julio de 2016, pp. 140-152. Recuperado de https://bit.ly/3IZYezA

CABRERA, F. J., CAPPELLO, M., GRECE, C. y VALAIS, S. (2016): VOD, platforms and OTT: which promotion obligations for European works? European Audiovisual Observatory. Recuperado de https://bit.ly/3oFHe3u

CALVO, A. G. (11 de marzo de 2021): “Nueve razones por las que 'The Wire' es una obra maestra". Sesnacine. Disponible en Internet: https://bit.ly/31P273a

CARTER, B. (1 de noviembre de 1992): "HBO as a Modern-Day Dickens". New York Times. Disponible en Internet: https://nyti.ms/3zRftau

CASCAJOSA, C.C. (2006): No es televisión, es HBO: La búsqueda de la diferencia como indicador de calidad en los dramas del canal HBO. ZER, Revista de Estudios de Comunicación, no21, noviembre de 2011, pp. 23-33. https://doi.org/10.1387/zer.3714

CELAYA, J. (2014): Nuevos modelos de negocio en la era digital, Madrid, Liber.

CBS News (26 de abril de 2002): "TV Guide Names Top 50 Shows". CBS News. Disponible en Internet: https://cbsn.ws/3CYivMl

DEFINO, D.J. (2014): The HBO Effect, Estados Unidos, Bloomsburry.

DEL PINO, C. y AGUADO, E. (2012): “Internet, Televisión y Convergencia: nuevas pantallas y plataformas de contenido audiovisual en la era digital. El caso del mercado audiovisual online en España". Observatorio (OBS) Journal, nำ4, 2012, pp. 57-75. https://doi.org/10.15847/obsOBS642012590

DURÁN MANSO, V. (2016): "La representación del deseo en el cine de Tennessee Williams: homosexualidad masculina frente al Código Hays". Femeris, n1-2, julio de 2016, pp. 58-73. http://dx.doi.org/10.20318/femeris.2016.3227

EDGERTON, G.R. y JONES, J.P. (2008): Essential HBO Reader, Estados Unidos, The University Press of Kentucky. 
El País (20 de mayo de 2019): “Así han respondido al final de 'Juego de Tronos' críticos de todo el mundo". El País. Disponible en Internet: https://bit.ly/3oeBgab

GARCÍA MARTínEZ, A.N. (2010): "La despedida más amarga ('In Treatment')". Diamantes en serie. Disponible en Internet: https://bit.ly/3zVL9eN

GUERRERO PÉREZ, E. (2011): "El ecosistema multiplataforma de los grupos televisivos españoles: los formatos de entretenimiento". Revista Comunicación y Hombre, no7, julio de 2011, pp. 85-103. Recuperado de https://bit.ly/3CSbigA

FERNÁNDEZ PENAS, M. y AGUADO PELÁEZ, D. (2013): “La hibridación como motriz de cambio en la las comedias de las series de televisión". Archivos de la Filmoteca, no72, enero de 2013, pp. 133-143. Recuperado de https://bit.ly/39JWzry

HUERTA, J.C. y DOMÍNGUEZ y SANZ, V. (2011): “Modelos over the top (OTT): regulación y competencia en los nuevos mercados de Internet". Política Económica y Regulatoria en Telecomunicaciones, no6, pp. 84-98.

HUERTAS PÉREZ, M. (2018): “Westworld a la estela de Juego de Tronos y de HBO”. Fòrum de recerca, $\quad \mathrm{n}$-13, 2018, pp. 115-128. http://dx.doi.org/10.6035/ForumRecerca.2018.23.8

IMDB (s.f.): "El show de Larry Sanders. Awards". IMDb. Disponible en Internet: https://imdb.to/3rYJIfx

IMDB (s.f.): "Los Soprano. Awards". IMDb. Disponible en Internet: https://imdb.to/3pVAcan

IZQUIERDO CASTILLO, J. (2012): “Distribución online de contenidos audiovisuales: análisis de los tres modelos de negocio". El profesional de la información, no4, julio de 2012, pp. 385-390. https://dx.doi.org/10.3145/epi.2012.jul.09

IZQUIERDO CASTILLO, J. (2015): “EI nuevo negocio mediático liderado por Netflix: estudio del modelo y proyección en el mercado español". El profesional de la información, no6, noviembre de 2015, pp. 819-826. https://doi.org/10.3145/epi.2015.nov.14

LASIERRA, I. (2012): "Nuevos caminos en las estrategias narrativas de una serie dramática de televisión: The Wire (HBO, 2002-2008)". Comunicación: revista Internacional de Comunicación Audiovisual, Publicidad y Estudios Culturales, no10, mayo de 2012, pp. 136-150. Recuperado de https://bit.ly/39JuHE2

LAVERY, D., HOWARD, D. L. y LEVINSON, P. (2011): The essential Sopranos Reader, Estados Unidos, The University Press of Kentucky. 
LEE, E. y KOBLIN, J. (8 de julio de 2018): "HBO Must Get Bigger and Broader, Says Its New Overseer". The New York Times. Disponible en Internet: https://nyti.ms/2XXOGOt

LEVERRETE, M., OTT, B.L. y BUCKLEY, C.L. (2008): It's Not T.V.: Watching HBO in the PostTelevision Age, Nueva York, Routledge.

LINARES BARRONES, Á. (2021): El viaje fragmentado del héroe. Su representación en la ficción televisiva Game of Thrones, Madrid, Fragua Editorial.

LOZANO DELMAR, J., RAYA BRAVO, I. y LÓPEZ RODRÍGUEZ, F. J. (2013): Reyes, espadas, cuervos y dragones. Estudio del fenómeno televisivo Game of Thrones, Madrid, Fragua Editorial.

MESCE, B. (2015): Inside the Rise of HBO, Estados Unidos, McFarland \& Company, Inc., Publishers.

MELLINS, M. (2012): "The Fantasia experience: True Blood fans, commodification and lifestyle", en CHERRY, B. (eds.): True Blood: Investigating Vampires and Southern Gothic. Investigating Cult TV Series, Londres, Tauris, pp. 172-185.

MONZONCILLO, J. M. y SENDRÁ, J. (2010): “La televisión, entre la gratuidad y el pago". Telos: Cuadernos de Comunicación e Innovación, n85, diciembre de 2010, pp. 3644. Recuperado de https://bit.ly/2Y4rn3x

OLIVEIRA JUNIOR, L. C. (2020): "Érase una vez en Westworld”. ARS (São Paulo), no38, abril de 2020, pp. 107-137. https://doi.org/10.11606/issn.21780447.ars.2020.165541

ONANDIA GARATE, M. (2013): "Tres obras maestras de la ficción televisiva: The Sopranos, The Wire y Mad Men". Revista del Departamento de Historia del Arte y Música de la Universidad del País Vasco, no3, julio de 2013, pp. 133-150. Recuperado de https://bit.ly/39R8CDw

OJER, T. y CANAPÉ, E. (2012): "Nuevos modelos de negocio en la distribución de contenidos audiovisuales: el caso de Netflix". Comunicación: revista Internacional de Comunicación Audiovisual, Publicidad y Estudios Culturales, №10, mayo de 2012, pp. 191-192. Recuperado de https://bit.ly/3F7LbUL

ORGAZ, C. (18 de diciembre de 2019): “Cómo AT\&T se convirtió en la empresa más endeudada del mundo (y qué tiene que ver HBO con ello)". BBC News. Disponible en Internet: $\underline{\text { https://bbc.in/3kO6BhE }}$

OSTERWALDER, A y PIGNEUR, Y. (2011): Generación de modelos de negocio, Barcelona, Deusto. 
PALLOTTA, F. (3 de octubre de 2019): “'Sesame Street' is heading to HBO Max in 2020". CNN. Disponible en Internet: https://cnn.it/3m8sBDg

PARSONS, P. (2003): "The evolution of the Cable-Satellite Distribution System". Journal of Broadcasting \& Electronic Media, no47, 2003, pp. 1-17. Recuperado de https://bit.ly/3CNjNJM

PEPPER, S. (2019): What "Sesame Street" Can Teach Us About HBO, Estados Unidos, Northeastern Illinois University.

PÉREZ MIRANDA, P. (30 de noviembre de 2021): "Último día para suscribirse a HBO Max a mitad de precio para siempre". El País. Disponible en Internet: https://bit.ly/3yaxfq8

POLAN, D. (2009): The Sopranos, Estados Unidos, Duke University Pres.

RUBIO HERNÁNDEZ, M. M. (2014): "Sexo en Nueva York". FRAME, no10, julio de 2014, pp. 111-115. Recuperado de https://bit.ly/2ZEh07r

SANTAELLA, F. (2016): "Series de HBO, piel del storytelling". Estudios Venezolanos de Comunicación, Centro Gumilla, no175, 2016, pp. 47-52. Recuperado de https://bit.ly/2WrujXN

SULLIVAN, J. (2010): Seven Dirty Words: The Life and Crimes of George Carlin. Estados Unidos, Da Capo Press Inc. Recuperado de https://bit.ly/2XWoh1r

ZARAZUA ABASCAL, D. (28 de septiembre de 2018): “HBO colgó los guantes después de 45 años de transmitir boxeo". AS. Disponible en Internet: https://bit.ly/2WhnTu4 